\title{
Condición física saludable y neurodidáctica: reflejo de una escuela inclusiva sostenible.
}

(Healthy and neurodidactic physical condition: reflection of a sustainable inclusive school.)

\author{
Cristina Marín Perabá \\ Universidad de Jaén (España) \\ cmp00058@ujaen.es \\ https://orcid.org/0000-0002-1329-9203
}

Fecha recepción:07/04/2021

Páginas 1-13

Fecha aceptación:11/05/2021

\section{Resumen.}

Este trabajo recoge la importancia de la neurodidáctica y la condición física saludable como facilitadoras de las escuelas inclusivas sostenibles. La investigación de condición no experimental, exploratoria, descriptiva y correlacional se enmarca en el objetivo de establecer el vínculo entre condición física saludable y neurodidáctica para promover la educación inclusiva y sostenible. En el estudio de metodología cuantitativa, se extrae información de una escala Likert realizada ad hoc para la recogida de información a una población de 300 estudiantes del Máster Universitario de Profesorado de Educación Secundaria Obligatoria de la Universidad de Jaén, de los cuales debido a las bajas, la muestra de la investigación será de 289. La escala Likert recoge un total de 32 ítems que han sido distribuidos entre las cuatro variables de estudio: Neurodidáctica, condición física saludable, escuela inclusiva y sostenibilidad. En la investigación se aprecia la inclusión escolar como aquella que tiene en cuenta las peculiaridades del alumnado y también, la neurodidáctica como potenciadora del funcionamiento del cerebro para el aprendizaje eficaz.

Palabras clave: actividad juvenil; alumno; deporte; educación especial; integración social

\section{Abstract.}

This work reflects the importance of neurodidactics and healthy physical condition as facilitators of sustainable inclusive schools. Non-experimental, exploratory, descriptive and correlational research is part of the objective of establishing the link between healthy and neurodidactic physical condition to promote inclusive and sustainable education. In the study of quantitative methodology, information is extracted from a Likert scale carried out ad hoc for the collection of information from a population of 300 students of the University Master's Degree in Compulsory Secondary Education Teachers of the University of Jaén, of which due to the low , the research sample will be 289. The Likert scale collects a total of 32 items that have been distributed among the four study variables: Neurodidactics, healthy physical condition, inclusive school and sustainability. In the research, school inclusion is appreciated as one that takes 
into account the peculiarities of the students and also neurodidactics as an enhancer of brain functioning for effective learning.

Keywords: social integration; special education; sport; student; youth activity

\section{1.-Introducción.}

Numerosos estudios describen que las problemáticas más relevantes en los entornos escolares son la escasez de actividad física y la carencia de inclusión (Urrea \& Rus, 2021 y Cobeñas, 2020).

Es función de los centros educativos contemplar las necesidades e intereses a nivel curricular de todos los alumnos del centro, y no sólo eso, también es su misión la de promover el continuo fortalecimiento del bienestar físico de los alumnos, ya que entre los problemas sociales se encuentra la obesidad infantil, y por tanto es un tema de interés a trabajar desde las instituciones educativas (Camargo \& Rodríguez, 2020).

Por otro lado, la inclusión sostenible en la escuela, es una cuestión que se lleva trabajando desde 1990 pero a día de hoy no se ha conseguido en su totalidad, por lo que es una materia aún pendiente en los centros escolares (Kem-Mekah, 2021).

Por estos y otros motivos relacionados con la calidad de educación, la neurodidáctica como unión de las neurociencias y la educación, facilita la comprensión de la actividad neuronal de los discentes a los profesores, con el fin de mejorar la atención individualizada en la escuela (Paniagua, 2013).

\section{1.-Neurodidática.}

Las neurociencias han ido avanzando con el paso de los años. En el año 1990, se inició un nuevo conocimiento acerca de la forma de trabajar en la educación, basándose en el análisis de las habilidades que tiene el cerebro, ya que en la investigación la educación y el cerebro son temas que han de ir de la mano, puesto que los avances que se hagan a nivel del cerebro han de influir directamente en la educación. De este nuevo conocimiento surgió la neurodidáctica en el que se usan las competencias del cerebro para dar al docente diferentes estrategias de enseñanza (Pinto, 2021).

Para Ramos y San Andrés (2019), la neurodidáctica es una rama de la pedagogía que se basa en las ciencias de las neuronas para orientar la educación. Su fin es buscar las estrategias y herramientas didácticas más eficaces para promover un mejor proceso de enseñanza-aprendizaje y por lo tanto, un mejor desarrollo del cerebro. Esto significa que el profesor o profesora, ha de ser capaz de crear las sinapsis suficientes, con sus respectivas conexiones neuronales, fomentando las conexiones cerebrales, siendo posible así la adecuada respuesta a la diversidad de los discentes, y por tanto la educación inclusiva (Briones, 2021). 
Gracias a la neurodidáctica se puede interpretar las actividades y conexiones que existen a nivel cerebral, con el fin de conocer si un alumno o alumna entiende las explicaciones del profesorado y las interioriza, o si por el contrario, nos escucha, pero su organización cerebral no le permite entender a sus docentes, ya que en la sociedad existe diversidad de organizaciones cerebrales (Paniagua, 2013).

\section{2.-Condición física saludable.}

En la historia del hombre, la actividad y la condición física siempre ha estado presente en todas las actividades las personas, ya que durante muchos siglos el estilo de vida incluía actividades físicas que le permitían subsistir. Sin embargo, con el paso de los años y los avances tecnológicos, la actividad física se ha visto mermada, surgiendo de aquí el sedentarismo o la inactividad física, lo que conlleva un aumento de mortalidad (Ries, 2008).

El concepto de salud, (entendiendo por salud aquello que no solamente es el ámbito psicológico y biológico, también se incluye el plano del bienestar y las condiciones sociales de las personas) siempre ha ido ligado a la realización de actividad física, por sus beneficios derivados de la práctica (Ries, 2008).

La promoción de una condición física saludable, es hoy en día una cuestión de interés para la sociedad, impulsando así los hábitos saludables de vida y la práctica de actividad física y/o deporte. Aspectos que son de fundamental interés en el ámbito educativo, puesto que la escuela es un espacio esencial comprometido con la prevención del sobrepeso y la obesidad, aportando así, una adecuada calidad de vida (Aguilar, Gil, Ortega \& Rodríguez, 2020). Siguiendo a Mora, García y Latorre (2017), la etapa de educación escolar es el mejor momento para la promoción de hábitos saludables, ya que el discente lo tomará como prácticas usuales para el resto de su vida.

Respecto a la condición física saludable durante la etapa escolar, Zamorano, Fernández y Vállez (2021), señalan que la práctica diaria de actividad física influye directamente en el rendimiento académico del alumnado, obteniendo beneficios después de la práctica de deporte en la atención, la memoria de trabajo, el control inhibitorio o mayor conexión de neuronas en el cerebro. Por lo contrario, si hay carencia de práctica física, se promueve el sedentarismo y en consecuencia, el rendimiento académico se ve reducido.

\section{3.-Inclusión escolar.}

La inclusión social se contempla como un proceso en el que las personas con riesgos 0 exclusiones sociales tengan las herramientas necesarias para participar en la vida social, económica y cultural (Alcaraz \& Arnaiz (2019); Gajardo \& Torrego, 2020).

En el ámbito de la inclusión, también se puede distinguir la inclusión educativa como aquello que surge de la exclusión y desigualdades educativas. Inclusión escolar, que 
ayuda a proporcionar una sociedad más desarrollada y justa, ya que la educación no es el único ámbito donde se ha de trabajar la inclusión, pero garantiza que las limitaciones de la educación sean eliminadas (Echeita \& Duk, 2008).

De acuerdo con Gajardo y Torrego (2020), la educación inclusiva se plantea para avalar el acceso a la educación de calidad en igualdad de oportunidades de todos los niños y niñas con o sin diversidad funcional. Para ello, se han de suprimir las barreras que impiden acceder a una educación de calidad, superando la discriminación y exclusión escolar. En definitiva, un colegio con prácticas inclusivas de calidad, es aquel que tiene en cuenta las necesidades educativas de todos sus discentes (Echeita \& Duk, 2008).

En relación con la actividad física en la escuela, Reina (2014) señala que cada alumno 0 alumna es diferente, con diversas capacidades y necesidades tanto físicas, como cognitivas, emocionales o sociales, por lo que todos los discentes tienen el mismo derecho a aprovecharse de las actividades físicas, independientemente de sus capacidades, aunque sí, suprimiendo aquellas barreras de diferentes índoles que dificultan el acceso a la actividad física inclusiva.

\section{4.-Sostenibilidad.}

Numerosos estudios muestran que desde hace siglos estamos llamados, la sociedad al completo, a la recuperación ambiental, ya que se están agotando los recursos, y por lo tanto estamos ante una llamada de emergencia por el medioambiente (KemMekah, 2021). Los análisis de Vilches, Gil y Cañal (2010) revelan una escasa atención a los problemas medioambientales a los que la sociedad no estamos enfrentando, sin tener en cuenta por lo tanto la opción del desarrollo sostenible.

El concepto de sostenibilidad no solamente se refiere a cuestiones de cuidados medioambientales con la ecología del planeta, también es una cuestión de cohesión social y convivencialidad, por lo que es un problema a resolver desde la educación (Iglesias, González-Patiño, Lalueza \& Esteban-Guitart, 2020).

La sostenibilidad apela a una sociedad justa e igualitaria, donde la educación sea repleta de diversidad de culturas, etnias, capacidades y/o habilidades, porque el cuidado del desarrollo sostenible es importante para la sociedad, pero más importante es el cuidado de las personas que lo componen (Iglesias et al., 2020).

\section{2.-Metodología.}

\section{1.-Material y método.}

El problema que se revela en esta investigación es el siguiente: ¿La condición física saludable y la neurodidáctica permiten desarrollar una escuela inclusiva sostenible? En el objetivo general se enmarca establecer el vínculo entre condición física 
saludable y neurodidáctica para promover la educación inclusiva y sostenible. De este objetivo principal, se suscitan nuevos objetivos específicos:

1.- Identificar las particularidades de la neurodidáctica que permiten hacer un aprendizaje significativo.

2.- Definir las características de la escuela inclusiva.

3.- Delimitar las características principales del desarrollo sostenible que hacen posible la inclusión en la educación.

4.- Analizar los diferentes factores existentes para una condición física saludable.

\section{2.-Diseño de investigación.}

Este trabajo de investigación se ha llevado a cabo mediante la recogida de datos a través del instrumento de escala Likert anónima, analizando posteriormente los mismos con el programa estadístico software SPSS v.25. La investigación es de condición no experimental, exploratoria, descriptiva y correlacional, y además, cuenta con una metodología cuantitativa.

\section{3.-Población y muestra.}

En esta investigación se ha tomado como participantes a una población de 300 estudiantes del Máster Universitario de Profesorado de Educación Secundaria Obligatoria y Bachillerato, Formación Profesional y Enseñanza de Idiomas. Estos alumnos, pertenecen a la Universidad de Jaén del curso 2020/2021, y de los cuales, teniendo en cuenta las ausencias, contamos finalmente con una muestra de 289 discentes, cuyos 61 son hombres y 228 son mujeres.

\section{4.-Instrumento.}

Para recoger los datos de la investigación, se diseñó una matriz de operacionalización aunando variables, ítems y unidades de medida (Mejía, 2005). Esta matriz de operacionalización fue finalmente utilizada para construir una escala Likert con un total de 32 ítems, distribuidos en cuatro variables, y comentadas a lo largo de esta investigación. Cada uno de los ítems han sido respondidos por nuestra muestra de discentes, mediante opciones de respuesta como: 1.- muy en desacuerdo, 2.- desacuerdo, 3.- indiferente, 4.- de acuerdo y 5.- muy de acuerdo (Alan y Atalay, 2020).

\section{5.-Dimensiones y variables.}

Para la elaboración de las dimensiones se llevó a cabo una revisión bibliográfica de las temáticas de interés, sirviendo como modelo para la elaboración de la escala 
Likert: A.- Neurodidáctica, B.- Inclusión educativa, C.- Sostenibilidad y D.- Condición física saludable.

Por consiguiente, las variables son de dos tipos:

-Variables independientes: Neurodidáctica y Condición física saludable

-Variables dependientes: Sostenibilidad e Inclusión educativa.

\section{6.-Procedimientos.}

El procedimiento de esta investigación se ha llevado mediante varias fases. En primer lugar se pidió un consentimiento a los responsables de la Universidad de Jaén para poder contar con la participación de nuestra muestra de investigación. Posteriormente, los sujetos de estudio firmaron un consentimiento informado en el que se incluían las bases y objeto de estudio, además de peligros y ventajas de la realización de la investigación. Finalizando con una validación del cuestionario mediante la valoración de un grupo de expertos, una prueba piloto, y un análisis factorial.

\section{3.-Resultados.}

\section{1.-Validez de contenido.}

En primer lugar, la validez de contenido se realizó por doctores autorizados de diferentes instituciones universitarias para llevar a cabo esta valoración (Malla y Zabala, 1978). Para éstos se calculó el Coeficiente de Conocimiento o Información (Kc) y el Coeficiente de Argumentación (Ka), y se siguió con el valor del Coeficiente de Competencia $(K)$ con la finalidad de determinar qué expertos doctores se tenían en cuenta para trabajar en dicho estudio, obteniendo un total de quince expertos con una $\mathrm{K}$ media de 0.9, lo que evidencia un alto nivel de competencia (López, 2011). Después de analizar los cuestionarios de validación, se realizó una prueba piloto a un grupo de la muestra para revisar aquellas dificultades que había en la comprensión del cuestionario, determinar las preguntas que generaban duda, etc., con lo que se utilizó la lista de control (Iraossi, 2006). Esta prueba piloto concurrió de forma adecuada ya que los resultados fueron satisfactorios y se dio por validado el instrumento en su contenido.

\section{2.-Fiabilidad.}

Para analizar la fiabilidad del instrumento se realiza el coeficiente de Alfa de Cronbach siguiendo a George y Mallery (2003), en el que se muestra una alta consistencia interna en el conjunto de los 32 ítems que se se presenta un valor de a $=0.8$, valor que se determina como excelente.

3.3.-Prueba de Kolmogorov-Smirnov. 
Para analizar la normalidad se selecciona la prueba de Kolmogorov-Smirnov, tras la cual se muestra que se debe rechazar la hipótesis nula, llegando a la conclusión de que los datos no siguen una distribución normal.

En efecto, se determina la siguiente hipótesis alternativa: H1.- El bienestar físico saludable con ayuda de la neurodidáctica desarrolla una escuela inclusiva sostenible.

\section{4.-Correlación de Spearman.}

Como no se cumple la normalidad de los datos, se utiliza la correlación Rho de Spearman.

Analizando los ítems del estudio, la correlación significativa (0.01) se da entre algunas de las siguientes variables más representativas de la investigación:

$\mathrm{A} 2 \rightarrow \mathrm{A} 6(0,70)$

A2.- Algunas de las claves de la neurodidáctica son: plasticidad cerebral, aprendizaje multisensorial, neuronas espejo, etc.

A6.- En la neurodidáctica, conocer el funcionamiento del cerebro de un/a alumno/a resulta útil para despertar el interés por aprender.

$$
\mathrm{B} 12 \rightarrow \mathrm{B} 15(0,58)
$$

B12.- La inclusión educativa implica la flexibilización de contenidos curriculares en caso de que sea necesario.

B15.- La formación continua en los/as profesores/as es uno de los elementos clave para una educación inclusiva de calidad.

$\mathrm{C} 22 \rightarrow \mathrm{C} 24(0,48)$

C22.- El desarrollo sostenible puede llevarse a cabo si se promueven acciones educativas inclusivas.

C24.- La sostenibilidad involucra la realización de acciones, teniendo en cuenta el compromiso, aprendizaje y conciencia de éstas.

$\mathrm{D} 27 \rightarrow \mathrm{D} 31(0,52)$

D27.- La condición física saludable se ve relacionada con las prácticas físicas diarias con el objetivo de desarrollar un bajo riesgo de enfermedades derivadas por el sedentarismo. 
D31.- Una buena condición física en los entornos escolares, se puede adquirir promoviendo actividades motoras en las que se involucre la participación y compromiso de todos los alumnos.

De la misma manera, se han obtenido otras correlaciones significativas entre diferentes dimensiones del estudio:

$A 6 \rightarrow D 25(0,56)$

A6.- En la neurodidáctica, conocer el funcionamiento del cerebro de un/a alumno/a resulta útil para despertar el interés por aprender.

D25.- La condición física saludable requiere tener energía vitalidad, hacer tareas diarias, ocio activo, etc.

En la anterior correlación entre estos dos ítems se determina que los sujetos investigados que señalan que en la neurodidáctica, conocer el funcionamiento del cerebro de un/a alumno/a resulta útil para despertar el interés por aprender, también indican además que la condición física saludable requiere tener energía vitalidad, hacer tareas diarias, ocio activo, etc.

$\mathrm{B} 9 \rightarrow \mathrm{A} 1(0,66)$

B9.- La inclusión educativa significa tener en cuenta las necesidades y peculiaridades de cada uno de los alumnos.

A1.- La neurodidáctica atiende las necesidades y potencial del funcionamiento del cerebro para un aprendizaje más eficaz.

Las personas encuestadas que están de acuerdo en que la inclusión educativa significa tener en cuenta las necesidades y peculiaridades de cada uno de los alumnos, son de la opinión de que la neurodidáctica atiende las necesidades y potencial del funcionamiento del cerebro para un aprendizaje más eficaz.

$\mathrm{C} 17 \rightarrow \mathrm{A} 4(0,59)$

C17.- La sostenibilidad implica atender los intereses y necesidades de las personas salvaguardando sus derechos.

A4.- La neurodidáctica en el aula ayuda al alumnado a aprender y a ser cada vez más autónomo/a.

En esta correlación, aquellos sujetos que opinan que en la sostenibilidad se tiene que tener en cuenta los intereses y necesidades de las personas salvaguardando sus derechos, también piensan que la neurodidáctica ayuda al discente a aprender y a más autónomo. 
$\mathrm{D} 30 \rightarrow \mathrm{B} 13(0,33)$

D30.- Para conseguir una buena condición física saludable en la escuela, hace falta que el alumnado sea participativo, que sea capaz de investigar o indagar, creando su propio aprendizaje como sujeto activo.

B13.- En la enseñanza inclusiva, se atiende a la construcción propia del aprendizaje incrementada con la interacción con los/las demás.

Aquellas personas que piensan que para conseguir una buena condición física saludable en la escuela, hace falta que el alumnado sea participativo, que sea capaz de investigar o indagar, creando su propio aprendizaje como sujeto activo, opinan además que en la enseñanza inclusiva, se atiende a la construcción propia del aprendizaje incrementada con la interacción con los/las demás.

\section{5.-Estadísticos descriptivos.}

Dimensión A (Neurodidáctica): Los sujetos participantes en este estudio están en desacuerdo en que la neurodidáctica es poco relevante para un aprendizaje significativo en el aula $(\bar{x}=2,06)$. Sin embargo, están de acuerdo en que el conocimiento por parte de los/as docentes sobre neurodidáctica para la buena práctica pedagógica, facilitará la sipnasis de redes neuronales del alumnado y por lo tanto se habrá conseguido una aprendizaje significativo $(\overline{\mathrm{X}}=4,07)$

Dimensión B (Inclusión educativa): Las personas investigadas responden de acuerdo en que la formación continua en los/as profesores/as es uno de los elementos clave para una educación inclusiva de calidad ( $\bar{x}=4,44)$. En cambio, están en desacuerdo en que en una educación inclusiva de calidad se puede prescindir del papel de la familia o de la sociedad, ya que solamente es necesaria la acción del docente y del resto de la comunidad educativa que concierne al centro educativo $(\bar{x}=1,53)$.

Dimensión C (Sostenibilidad): Los estudiantes encuestados responden de acuerdo en la sostenibilidad involucra la realización de acciones, teniendo en cuenta el compromiso, aprendizaje y conciencia de éstas $(\bar{X}=4,06)$. Pero a pesar de ello, se muestran indiferentes ante que la sostenibilidad requiere ser tolerante, pacífico y justo, pero no se tiene tanto en cuenta el papel de la inclusión $(\bar{x}=2,65)$.

Dimensión $D$ (Condición física saludable): Las personas participantes están de acuerdo en que la condición física saludable es necesaria para un buen rendimiento escolar ( $\bar{x}=4,09)$, así como en que la condición física saludable se ve relacionada con las prácticas físicas diarias con el objetivo de desarrollar un bajo riesgo de enfermedades derivadas por el sedentarismo $(\overline{\mathrm{X}}=4,20)$.

\section{4.-Discusión.}


La investigación que aquí se muestra, se llevó a cabo en una muestra de 289 alumnos del Máster de Profesorado de la Universidad de Jaén. Los resultados recogidos sirven como apertura para seguir indagando en el campo de la neurodidáctica, bienestar, inclusión y sostenibilidad, con lo que este estudio está abierto a cambios con el fin de seguir indagando para mejorar la intervención y la atención al alumnado con diversidad funcional. Por consiguiente, sería conveniente realizar esta misma investigación con alumnado de otros másteres relacionados con la educación, con alumnado de grado de Educación Primaria o Infantil, e incluso entre otras universidades, con el fin de recoger resultados en diferentes niveles y grados de las universidades, para posteriormente correlacionarlos entre sí, haciendo de la misma manera un estudio de validación con el fin de confirmar los factores mediante un análisis factorial confirmatorio, en el que se usará el instrumento realizado para la población de máster de Profesorado.

Por su parte, la inclusión escolar sostenible con alumnado con condición física saludable aún no está implantada en su totalidad en la sociedad, es por ello que la neurodidáctica servirá de apoyo para fomentar ambientes agradables de participación en cooperación y colaboración en el aula, atendiendo las necesidades e intereses de cada uno de los estudiantes (Alsia, Mallol y Alsina, 2020). De aquí la importancia de concienciar a la sociedad y aportar conocimiento que sea de utilidad para todas las personas, con el fin de conseguir una inclusión sostenible escolar plena, pensada y preparada para todas las personas. Existen estudios científicos que ratifican la necesidad de elaborar instrumentos de medición con carácter cuantitativo de investigación, que permitirán evaluar la inclusión sostenible mediante la neurodidáctica y la condición física saludable en los ámbitos educativos (Chandler y Greene, 1995).

\section{5.-Conclusión.}

En la presente investigación el objetivo principal que se ha concretado ha sido establecer el vínculo entre condición física saludable y neurodidáctica para promover la educación inclusiva y sostenible, teniendo presentes las dimensiones neurodidáctica, inclusión escolar, sostenibilidad y condición física saludable. Esta investigación se llevó a cabo en una muestra de alumnado universitario del máster jiennense de Profesorado de Educación Secundaria Obligatoria del curso académico 2020/2021. Para alzancar este fin, se procede a la elaboración de un instrumento Likert mediante una tabla de operacionalización. Se lleva a cabo el análisis de fiabilidad con el de Alfa de Cronbach $(\alpha=0.8)$, y posteriormente, tras comprobar con la prueba de Kolmogorov-Smirnov que se rechaza la hipótesis nula, se calcula la correlación Rho de Spearman, y por consiguiente, se destacan relaciones relevantes como la correlación $\mathrm{B} 9 \rightarrow \mathrm{A} 1(0,66)$ en el que se piensa que la inclusión educativa significa tener en cuenta las necesidades y peculiaridades de cada uno de los alumnos y que por lo tanto, también la neurodidáctica atiende las necesidades y potencial del funcionamiento del cerebro para un aprendizaje más eficaz. Por otro lado, los sujetos participantes en este estudio están en desacuerdo en que la 
neurodidáctica es poco relevante para un aprendizaje significativo en el aula, con lo cual, los resultados obtenidos, apoyan los datos de la investigación aquí presentada.

\section{6.-Referencias.}

Aguilar Jurado, M. A., Gil Madrona, P., Ortega Dato, J. F., \& Rodríguez Blanco, Ó. F. (2020). Mejora de la condición física y la salud en estudiantes tras un programa de descansos activos. Revista Española de Salud Pública, 92, 110.

Alan, Ü. \& Atalay, K. (2020). Effect of number of response option on the psychometric properties of Likert-type scales used witch children. Studies in Educational Evaluation, 66, 1-8. https://doi.org/10.1016/i.stueduc.2020.100895

Alcaraz, S. \& Arnaiz, P. (2019). La escolarización del alumnado con necesidades educativas especiales en España: un estudio longitudinal. Revista Colombiana de Educación, 1(78), 299-320. https://doi.org/10.17227/rce.num78-10357

Alsina, M., Mallol, C. \& Alsina, A. (2020). El reto inclusivo desde la educación artística transformativa. En L. Habib-Mireles (ed.). Tecnología, diversidad e inclusión: repensando el modelo educativo (pp. 114-125). Adaya Press.

Briones, G. (2021). Estrategias neurodidácticas en el proceso de enseñanzaaprendizaje de educación básica. ReHuSo: Revista de Ciencias Humanísticas y Sociales, 6(1), 56-64.

Camargo, D. A., \& Rodríguez, M. A. (2020). Condición física e indicadores de riesgo cardiovascular en población con discapacidad intelectual. Revista Ciencias de la Salud, 18(1),

$10-$

24. https://doi.org/10.12804/revistas.urosario.edu.co/revsalud/a.8736

Cobeñas, P. (2020). Exclusión educativa de personas con discapacidad: Un problema pedagógico. REICE. Revista Iberoamericana sobre Calidad, Eficacia y Cambio en Educación, 18(1), 65-81. https://doi.org/10.15366/reice2020.18.1.004

Chandler, J.P. \& Greene, J.L. (1995). A statewide survey of adapted physical education service delivery and teacher in-service training. Adapted Physical Activity Quarterly, 12(3), 262-274. https://doi.org/10.1123/apaq.12.3.262

Echeita, G. \& Duk, C. (2008). Inclusión educativa. REICE, 6(2), 1-8.

Gajardo, K., \& Torrego, L. (2020). Representaciones sociales sobre inclusión educativa de una nueva generación docente. Revista Educación, política y sociedad, 5(1), 11-38. 
George, D., \& Mallery, P. (eds.) (2003). SPSS for Windows step by step: A simple guide and reference. 11.0 update (4thed.). Allyn \& Bacon

Iglesias, E., González-Patiño, J., Lalueza, J. L., \& Esteban-Guitart, M. (2020). Manifiesto en tiempos de pandemia: Por una educación crítica, intergeneracional, sostenible y comunitaria. Revista Internacional de Educación para la Justicia Social, 9(3), 181-198. https://doi.org/10.15366/riejs2020.9.3.010

Iraossi, G. (eds.) (2006). The power of survey desing: A user's guide for managing surveys, interpreting results, and influencing respondents. The World Bank. https://doi.org/10.1596/978-0-8213-6392-8

Kem-Mekah, O. (2021). Retos para una educación sostenible e inclusiva en el siglo $\mathrm{XXI}$. Experiencias singulares. SUMMA. Revista disciplinaria en ciencias económicas y sociales, 3(1), 1-16. https://doi.org/10.47666/summa.3.1.06

López, R. (2011). Bases conceptuales de la educación inclusiva. Avances en Supervisión Educativa, (14), 1-20.

Malla, F. \& Zabala, I. (1978). La previsión del futuro en la empresa (III). El método Delphi. Estudios Empresariales, 39(1), 13-24.

Mejía, E. (2005). Metodología de la investigación científica. Universidad Nacional Mayor de San Marcos

Mora, D. J., García, F. \& Latorre, P. A. (2017). Actividad física, condición física y salud en niños preescolares. Estudio de revisión narrativa. Revista digital de Educación Física, 8(45), 105-123.

Paniagua, M. N. (2013). Neurodidáctica : una nueva forma de hacer educación. Fides et Ratio, 6(6), 72-77.

Pinto, C. (2021). La neurociencia para la inclusión en contextos pluriculturales. Revista Internacional de apoyo a la inclusión, logopedia, sociedad y multiculturalidad, 7(1), 1-8. https://doi.org/10.17561/riai.v7.n1.1

Ramos, A. M. \& San Andrés, E. M. (2019). Neurodidáctica y competencias emocionales de estudiantes de educación general básica. Revista Interdisciplinaria de Humanidades, Educación, Ciencia y Tecnología, 6(1), 1629. https://doi.org/10.35381/cm.v5i1.237

Reina, R. (2014). Inclusión en deporte adaptado: dos caras de una misma moneda. Psychology, Society, \& Education, 6(1), 55-67. 
Ries, F. (2008). Estudio sobre la condición física saludable: una revisión bibliográfica hasta el año 2005. Revista Fuentes, 8, 299-321.

Urrea, A. S., \& Rus, T. I. (2021). Factores socioeconómicos que influyen en la salud nutricional y actividad física de escolares. Retos: nuevas tendencias en educación física, deporte y recreación, (40), 95-108.

Vilches, A., Gil, D. \& Cañal, P. (2010). Educación para la sostenibilidad y educación ambiental. Investigación en la escuela, (71), 5-15. https://doi.org/10.12795/IE.2010.i71.01

Zamorano, D., Fernández, J. G. \& Vállez, J. P. (2021). Diseño y evaluación de un programa para la mejora de la condición física en Educación Primaria: efecto sobre la autoestima y la intención de ser activo. SPORT TK-Revista EuroAmericana de Ciencias del Deporte, 10(1), 107-112. https://doi.org/10.6018/sportk.461721 